\title{
Optimal control of brakes and steering for autonomous collision avoidance using Modified Hamiltonian Algorithm
}

\author{
Yangyan Gao ${ }^{\mathrm{a} *}$, Timothy Gordon ${ }^{\mathrm{a}}$ and Mathias Lidberg ${ }^{\mathrm{b}}$ \\ ${ }^{a}$ School of Engineering, University of Lincoln, Brayford Pool, Lincoln LN6 7TS, UK; ${ }^{b}$ Applied \\ Mechanics, Chalmers University of Technology, SE-412 96 Gothenburg, Sweden
}

(Received 00 Month 20XX; accepted 00 Month 20XX)

\begin{abstract}
This paper considers the problem of collision avoidance for road vehicles, operating at the limits of friction. A two-level modelling and control methodology is proposed, with the upper level using a friction-limited particle model for motion planning, and the lower level using a nonlinear 3DOF model for optimal control allocation. Motion planning adopts a two-phase approach: the first phase is to avoid the obstacle, the second is to recover lane keeping with minimal additional lateral deviation. This methodology differs from the more standard approach of path-planning/path-following, as there is no explicit path reference used; the control reference is a target acceleration vector which simultaneously induces changes in direction and speed. The lower level control distributes vehicle targets to the brake and steer actuators via a new and efficient method, the Modified Hamiltonian Algorithm (MHA). MHA balances CG acceleration targets with yaw moment tracking to preserve lateral stability. A nonlinear 7DOF two-track vehicle model confirms the overall validity of this novel methodology for collision avoidance.
\end{abstract}

Keywords: Vehicle dynamics, Vehicle control, Collision avoidance, Active safety, Stability control

\section{Introduction}

The development of Collision Avoidance systems has undergone a transition from warning systems, where the driver is expected to respond quickly to a visual or audible alert [1-3], towards auto-braking systems such as $[4,5]$. There appear to be clear safety benefits from autonomous emergency braking systems although such systems are normally restricted to operate at lower speeds $[5,6]$.

Studies on collision avoidance have clearly indicated that braking is more efficient at lower speeds and steering is more efficient at higher speeds [7]. However, it is far from clear that pure braking or pure steering always provides the most efficient form of collision avoidance; combined braking and steering may be more effective over a range of conditions of speed, range and required lateral deviation. Hence, in this paper, we will investigate various combinations of steering and braking to achieve collision avoidance using a common design framework. A typical accident avoidance scenario has a stationary vehicle ahead of the subject vehicle, at a range which is too small for pure braking to avoid a collision; steering to avoid an impact remains possible but the driver fails to respond. Then, to avoid collision, an emergency lane change is planned and executed 
autonomously. Here we do not consider the possibility of collisions with other vehicles due to the lane change manoeuvre, presuming that such risks are very low. While we assume 'steer to avoid' is required, we allow for the possibility of simultaneous braking action providing assistance in the maneuver.

In existing literature, it is typical to adopt a two-level strategy of path planning followed by path tracking $[5,7,8]$. There are a number of limitations in terms of optimality, complexity, or over-reliance on an accurate vehicle model which makes the real world implementation challenging and, in most cases, infeasible. Two examples illustrate the points. In [8], Nonlinear Model Predictive Control (NMPC) is used to avoid obstacles and keep the vehicle in the lane; in [5] a framework is implemented combining model predictive and feedback control to target path tracking, vehicle stabilization, and collision avoidance objectives in a coordinated manner. The first approach can lead to infeasible or over-conservative path planning (due to the highly dynamic nature of the situation) while the second approach relies on precise gain tuning for a specific test vehicle.

There remains a need for a controller design method that avoids the complexity of NMPC in real-time, uses a minimal number of tuning parameters, and does not artificially separate the path and speed planning from dynamic motion control.

Other methods have been explored using numerical optimization to avoid the need for a predefined collision-free path. In [9], the avoidance problem is formulated as minimization of the total vehicle force using a point-mass vehicle model under a friction constraint. The overall methodology is somewhat similar to that used below, the upperlevel optimal control problem being reduced in complexity via a particle assumption. However, this work used a rather complex tyre-force allocation scheme to distribute the calculated global vehicle level forces into individual longitudinal and lateral tyre forces. A hierarchical control structure was adopted and the distributing algorithm used Sequential Quadratic Programming (SQP). The complexity of the overall control structure makes real-time implementation unrealistic, particularly since the optimization is not guaranteed to converge.

Previously, a novel control method termed the Modified Hamiltonian Algorithm (MHA) [10-12] was developed for an Autonomous Emergency Cornering system to prevent highway vehicles from dangerous lane departure due to excessive speed. The underlying control concept has been tested with an experimental vehicle and the results suggest that MHA can run efficiently in real time with a highly satisfactory control performance [11]. In the present paper, a Collision Avoidance (CA) system is developed using a similar general approach. Due to the highly transient nature of the current problem, MHA requires an effective yaw moment control strategy combined with optimal path and speed control.

The paper is structured as follows: in section 2, a number of collision avoidance strategies are compared using a friction-limited particle model of the vehicle, leading to an optimal control formulation. Section 3 introduces the MHA method. Then the upper and lower level controllers are combined in simulation in section 4. Section 5 concludes the paper and discusses the possibilities for future implementation.

\section{Particle Reference}

As mentioned, the overall vehicle controller is designed with a hierarchical structure. Here we consider the upper level control, which uses a particle model to approximate an optimal solution. Hence a target mass-center acceleration reference is obtained. The 
particle motion is defined via a friction-limited acceleration vector,

$$
\mathbf{a}(t)=\ddot{X}_{E} \mathbf{i}+\ddot{Y}_{E} \mathbf{j} \quad \sqrt{\ddot{X}_{E}^{2}+\ddot{Y}_{E}^{2}} \leq \mu g
$$

assuming a circular friction limit. Then in the vehicle application, this represents the target mass centre acceleration

$$
\mathbf{a}^{d}=\mathbf{a}(t)
$$

When motion takes place on the friction limit, the equations of motion are:

$$
\begin{aligned}
& \ddot{X}_{E}=\mu g(\cos \theta) \\
& \ddot{Y}_{E}=\mu g(\sin \theta)
\end{aligned}
$$

and the angle $\theta(t)$ of the acceleration vector is then a scalar control variable with respect to the global $X$ coordinate, see Figure 1.

\subsection{Simple strategies}

In Figure 1 we present a conceptual solution to the optimal collision avoidance problem which includes two phases of control. The first phase $\left(\mathrm{P}_{0}\right.$ to $\left.\mathrm{P}_{1}\right)$ is to avoid the collision, and the second $\left(\mathrm{P}_{1}\right.$ to $\left.\mathrm{P}_{2}\right)$ is to minimize 'over-shoot' $\Delta y$, i.e. intrusion into the adjacent lane. Parameter $A$ is the available distance for braking or steering to avoid the obstacle, and $B$ is the required lateral deviation for avoidance. The particle path may be identified with a target CG trajectory of the vehicle, though as mentioned it is only the acceleration profile that is tracked during vehicle control. Note that the collision avoidance condition at $\mathrm{P}_{1}$ should make allowance for vehicle width (plus any 'safety margin' distance). We then require that the vehicle body sideslip angle $\beta$ remains small, and this condition forms part of the (MHA) control allocation given below.

It is easy to show that in the second phase $\left(\right.$ after $\left.\mathrm{P}_{1}\right)$ a constant acceleration with $\theta=-90^{\circ}$ is required. In that case, $\Delta y=v_{\perp}^{2} /(2 a)$, where $v_{\perp}$ is the track-lateral component of velocity at $\mathrm{P}_{1}$ and $a$ is the magnitude of resultant acceleration vector $\mathbf{a}^{d}$-see Equation 2. As shown in Figure 1, the acceleration vector may rotate during the first (collision

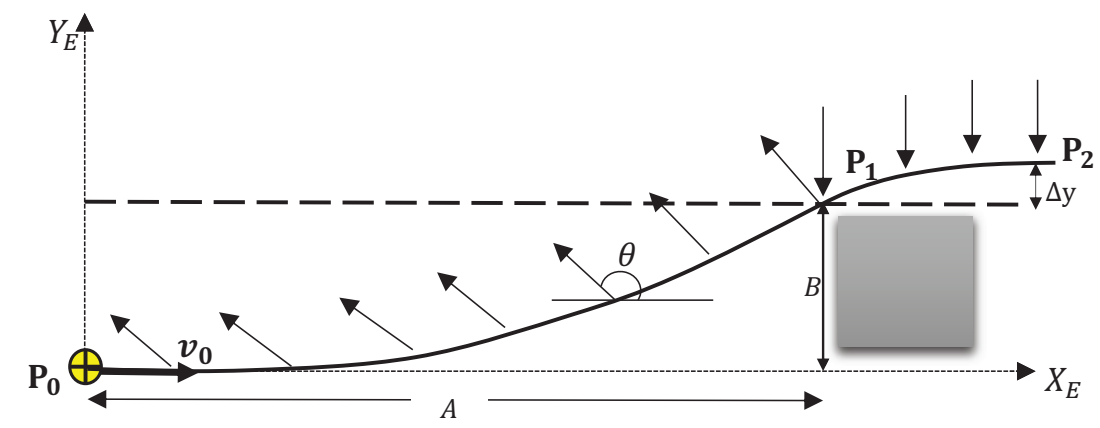

Figure 1. Concept and nomenclature for planar motion with two phase control for collision avoidance. The position and dimension of the obstacle is shown as a grey box.

avoidance) phase and, in the following, we will find this is typical for the optimal control. 
However, for comparison we will consider certain special cases, including where $\theta$ is constant during this phase, in which case:

$$
\begin{aligned}
A & =v_{0} t+\frac{1}{2} a(\cos \theta) t^{2} \\
B & =\frac{1}{2} a(\sin \theta) t^{2}
\end{aligned}
$$

The simplest situation is $\theta=180^{\circ}$ corresponding to straight-line braking (SLB). The over-shoot is then zero and the minimum friction-limited braking distance is $A_{S L B}=$ $v_{0}^{2} /(2 a)$. A steering-biased collision avoidance strategy is with track-lateral acceleration (TLA), i.e. $\theta=90^{\circ}$ and $A_{T L A}=\sqrt{2 B v_{0}^{2} / a}$ is the minimum distance for the steering avoidance phase. The TLA strategy involves a slight increase in vehicle speed, so a more realistic reference for 'steer to avoid' is path-lateral acceleration (PLA), where initially $\theta=90^{\circ}$, then the acceleration vector rotates to remain perpendicular to the particle trajectory. The resulting path is a circular arc of radius $R=v_{0}^{2} / a$, and the minimum distance is easily determined as $A_{P L A}=\sqrt{2 B R-B^{2}}$. Sample results are given in Table 1 .

Table 1. Braking distance and lateral over-shoot for three different collision avoidance strategies with parameters: $\mu=0.9, v_{0}=30 \mathrm{~m} / \mathrm{s}, B=3.8 \mathrm{~m}$

\begin{tabular}{|l||l|l|l|}
\hline & $S L B$ & $T L A$ & $P L A$ \\
\hline$A(\mathrm{~m})$ & 51.97 & 27.93 & 27.57 \\
$\Delta y(\mathrm{~m})$ & 0 & 3.8 & 3.73 \\
\hline
\end{tabular}

The (well-known) conclusion is that collision-avoidance by steering can be effective when the available distance is too short for pure braking. However, the lateral motion leads to over-shoot, and the 'price to pay' for the shorter avoidance distance is the extra lateral deviation, equivalent to an additional lane-width $(3.8 \mathrm{~m})$ for TLA. The PLA strategy is seen to be slightly better in both aspects, presumably because there is no increase in speed. In the next part we consider the optimal particle strategy.

\subsection{Optimal control for a particle representation}

We start from the equations of motion for the friction-limited particle model - Equations 1, 3. For given distances $A$ and $B$, the objective is to minimize over-shoot $\Delta y$ - see Figure 1. For a non-trivial solution, we focus on the case where $A$ is large enough to avoid collision (assume $v_{0}$ and $B$ are fixed), but insufficient to achieve $\Delta y=0$ either by straight-line braking or some other strategies.

We now re-state the optimization problem: to minimize lateral off-tracking into the adjacent lane $\left(\Delta y=Y_{E \mathrm{P}_{2}}-B\right)$ subject to the constraint of avoiding collision at $\mathrm{P}_{1}$ :

$$
\begin{gathered}
\min _{\mathbf{a}(t)} \Delta y \\
y\left(t_{1}\right) \geq 0
\end{gathered}
$$

where $t_{1}$ is the time at point $\mathrm{P}_{1}$. However, between $\mathrm{P}_{1}$ and $\mathrm{P}_{2}$ the optimal control is simply $\mathbf{a}=[0,-\mu g]^{\mathrm{T}}$, and hence $\Delta y=\dot{y}\left(t_{1}\right)^{2} / 2 \mu g$. Further, for this type of problem the control will remain at its limits [13]. Hence the optimization can be restricted to phase 1 and the optimality condition - Equation 5 is simplified to the minimization of a terminal 


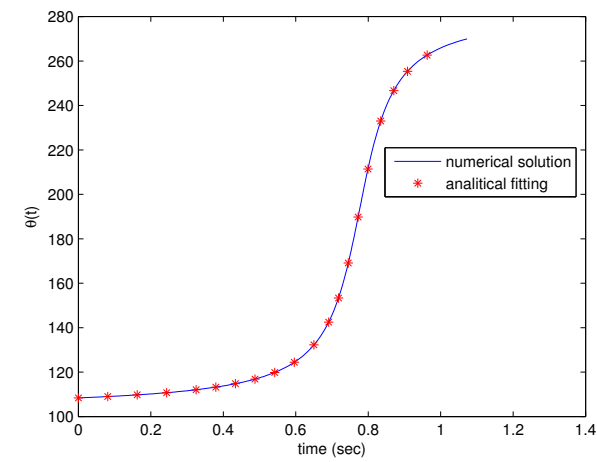

Figure 2. Mass centre acceleration reference under optimal control: blue solid line represents numerical solution; red star obtained from analytical solution Equation 7.

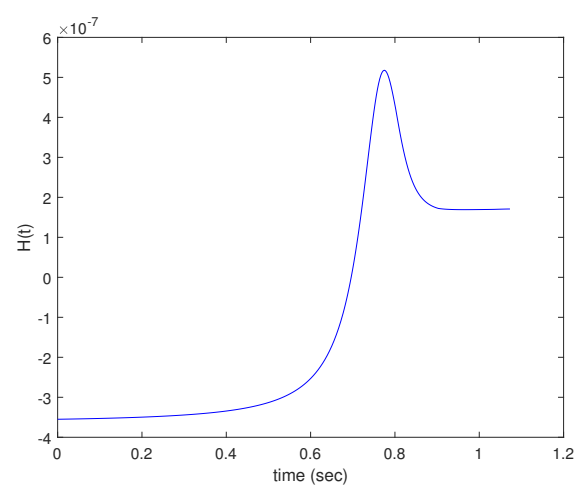

Figure 3. The value of Hamiltonian function under optimal control.

cost $\dot{y}\left(t_{1}\right)$ at $\mathrm{P}_{1}$ with $\theta$ as the control variable:

$$
J=\min _{\theta} \dot{y}\left(t_{1}\right)
$$

This optimal control problem is solved in the Appendix A, where the optimal control can be obtained by solving the coupled state and costate equations as a two-point boundary value problem. The optimal control law is found to be in the form of a 'bilinear tangent law' [13]:

$$
\tan \theta^{*}(t)=\frac{k_{3} t+k_{4}}{k_{1} t+k_{2}}
$$

Constants $k_{1}, k_{2}, k_{3}, k_{4}$ are determined, though not fully explicitly, in terms of the problem parameters. Here we solve the optimization problem numerically and use the analytical solution as a check on accuracy. We apply the MATLAB built-in function bvp4c [14] for solving the two-point-boundary-value problem given in the Appendix A. Figure 2 show the numerical solution (blue line), and is compared to analytical solution obtained from Equation 7 (red stars). Figure 3 gives further validation of the solution, as the Hamiltonian function should equate to zero (see Appendix).

In the following, the particle reference is pre-computed and used as a fixed reference. In real-world applications an updating reference will be required and the formulation of the Appendix A may be used to implement this - for example by creating a lookup table relating parameters $k_{i}$ to the updating geometric parameters of the collision avoidance problem. This 'updating reference' controller is not considered further in this paper.

\subsection{Constant $\theta$ optimization}

It is found that as distance $A$ approaches the limit for the above numerical optimization to converge, (slightly less than for the simple steer strategies mentioned in section 2.1) the duration of the period of rotation becomes very small, i.e. the optimal particle acceleration approximates to $\theta=$ constant. It is therefore worth considering the problem of optimizing $\theta$ as a constant control parameter, and then compare with the more general case. 
From Equation 4, eliminating $t$

$$
A-B(\tan \theta)=v_{0} \sqrt{\frac{2 B}{a(\cos \theta)}}
$$

To find the minimum value of $A$, differentiate with respect to $\theta$ and impose $d A / d \theta=0$; we obtain:

$$
(\cos \theta)(\sin \theta)^{2}=\frac{2 B a}{v_{0}^{2}}
$$

which can be solved numerically for $\theta$. It is convenient to introduce the dimensionless parameter $\alpha=2 B a / v_{0}^{2}$. Once the optimal value of $\theta$ is found from Equation 9, the resulting (minimum crash avoidance) distance is obtained:

$$
A^{*}=\left(\frac{2}{\sqrt{\alpha(\cos \theta)}}+(\tan \theta)\right) B
$$

For the sample case of Table 1 we find $\theta^{*}=1.8688 \mathrm{rad}$, and $A^{*}=25.84 \mathrm{~m}$, i.e. slightly less than for the simple steering strategies and much less than for SLB.

\subsection{Multi-objective performance trade-off}

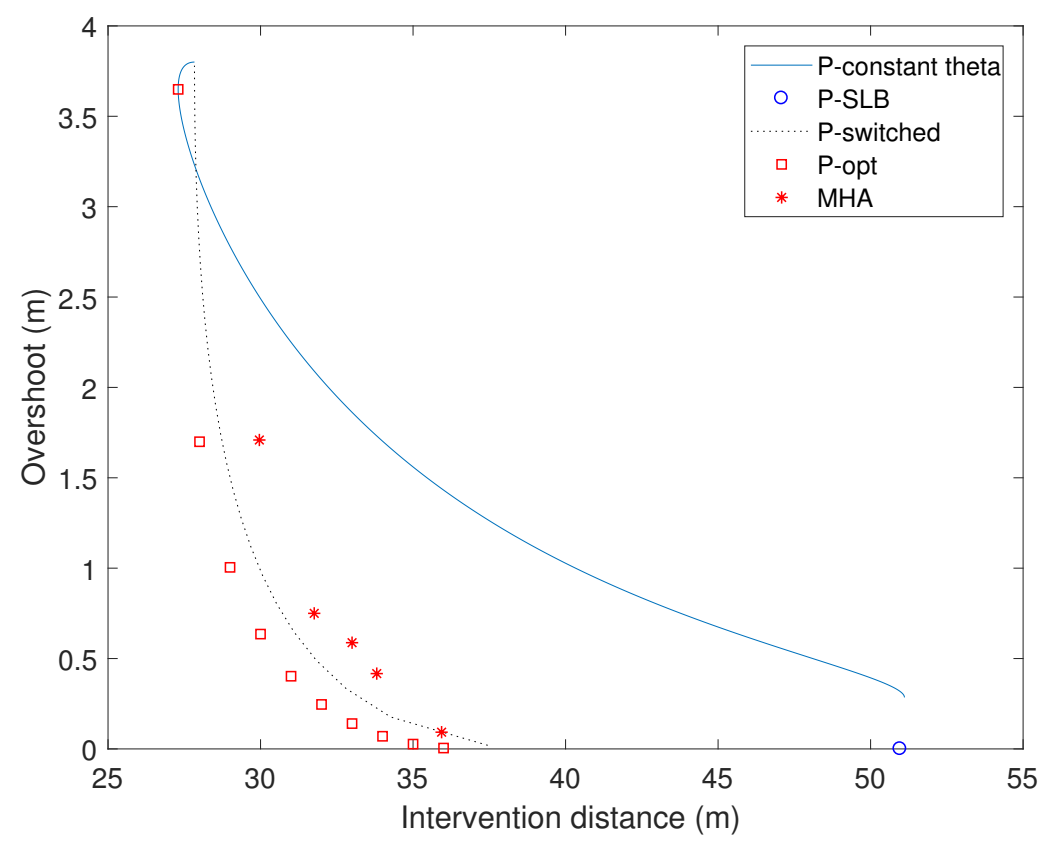

Figure 4. Trade-off between over-shoot $\Delta y$ and intervention distance $A$ for several strategies. The red squares represent the Pareto front, obtained by the optimal control method of section 2.2. P-refers to particle results, MHA refers to vehicle simulation (section 4). No solution is possible for $A<27.3 \mathrm{~m}$.

The optimization problem has been formulated, to minimize the over-shoot $\Delta y$ for given $A$. According to Table 1, if $A$ is larger than around $52 \mathrm{~m}$ (for the parameters shown) 
then no over-shoot is required. At shorter distances it is necessary to perform the lateral manoeuvre and some over-shoot may be necessary. A number of results are plotted in Figure 4. The solid blue line is obtained from Equation 4, where $\theta=$ constant is varied to define a simple trade-off. The blue circle is for straight-line braking (single point) and the dashed black curve is for a switching strategy during the first phase, $\theta=270^{\circ}$ for $t>t_{s}$. The switching time $t_{s}$ is varied to produce the curve. Optimal control results are plotted as red squares - these represent the Pareto front of multi - objective optimization, i.e. $\Delta y$ can only be reduced by increasing $A$. As $A$ tends towards its minimum, the optimal control results tend towards a solution with $\theta=$ constant; hence the uppermost square coincides with the $\theta=$ constant (blue curve). Close to the minimum distance for collision avoidance, $\Delta y$ becomes very sensitive to $A$, i.e. the unavoidable over-shoot rises very quickly as the available intervention distance reduces. On the other hand, with $A \geq 36 \mathrm{~m}$, both the optimal and switching strategies give negligible over-shoot. This is also true for the vehicle implementation (MHA) described in the following section.

\section{Vehicle Implementation Using MHA}

\subsection{Vehicle and Tyre Model}

We introduce a nonlinear vehicle model for simulation, a 7-degree-of-freedom (DOF) planar two-track model with three motion states $\left(v_{x}, v_{y}, \dot{\psi}\right)$ relative to the vehicle body coordinates $(x, y)$, plus four wheel rotational speeds $\dot{\omega}_{i}$. Wheel indices $i=(1,2,3,4)$ correspond to front-left, front-right, rear-left, rear-right locations respectively. While suspension motion is not fully represented, a load transfer model is used, including the effects of the roll-moment ratio $\Lambda$ between front and rear suspensions. The aim is to include the major dynamic processes relevant to non-linear transient dynamics, without making the model overly complex. The equations of motion are:

$$
\begin{aligned}
& M\left(\dot{v}_{x}-v_{y} \dot{\psi}\right)=F_{x 1}+F_{x 2}+F_{x 3}+F_{x 4} \\
& M\left(\dot{v}_{y}+v_{x} \dot{\psi}\right)=F_{y 1}+F_{y 2}+F_{y 3}+F_{y 4} \\
& I_{z} \ddot{\psi}=l_{f}\left(F_{y 1}+F_{y 2}\right)-l_{r}\left(F_{y 3}+F_{y 4}\right)+\mathrm{w} / 2\left(F_{x 2}-F_{x 1}\right)+\mathrm{w} / 2\left(F_{x 4}-F_{x 3}\right) \\
& \dot{\omega}_{i}=I_{w}^{-1}\left(T_{i}-R_{w} F_{x i}^{t}\right)
\end{aligned}
$$

Here $\left(F_{x i}, F_{y i}\right)$ are the longitudinal and lateral tyre forces resolved in vehicle body axes, $T_{i}$ are the wheel drive torques (if positive) or brake torques (if negative), and $I_{w}$ is the nominal wheel inertia, assumed equal for all wheels. Other parameters are the wheel rolling radius $R_{w}$, distances $\left(l_{a}, l_{b}\right)$ from the mass centre to the front and rear axles respectively, and $\mathrm{w}$ is the track width of the vehicle.

Forces $\left(F_{x i}, F_{y i}\right)$ are related to the components $\left(F_{x i}^{t}, F_{y i}^{t}\right)$ in tyre axes via the steering angle:

$$
\begin{array}{ll}
F_{x 1,2}=F_{x 1,2}^{t} \cos \delta-F_{y 1,2}^{t} \sin \delta & F_{x 3,4}=F_{x 3,4}^{t} \\
F_{y 1,2}=F_{y 1,2}^{t} \cos \delta-F_{x 1,2}^{t} \sin \delta & F_{y 3,4}=F_{y 3,4}^{t}
\end{array}
$$

Tyre forces $\left(F_{x i}^{t}, F_{y i}^{t}\right)$ are modelled via a standard Pacejka magic tyre (MF) formula, see Equation 13, which is a load-dependent combined-slip model, using normalized slip and isotropic similarity scaling $[15,16]$. Further details of the MF model are given in $[10,17]$. 


$$
P(x)=D \sin \left(C \tan ^{-1}\left(B x-E\left(B x-\tan ^{-1} B x\right)\right)\right)
$$

where $x$ is the input variable: slip angle $\alpha$ or slip ratio $s_{x}$ and $P$ is the output variable: longitudinal force $F_{x}$ or lateral force $F_{y}$.

The above tyre model is only a broad representative of real tyre behaviour, but is thought to incorporate sufficiently realistic aspects of force generation from the linear region up to saturation. The MHA controller also comprises the same tyre model for commanded tyre force generation. Some of the key vehicle and tyre parameters are given in Table 2. To further implement the MHA controller with a high-fidelity vehicle model or an experimental vehicle, this tyre model can be easily tuned (with only two key parameters $B$ and $C$ ) to fit to that of the simulation vehicle model or test vehicle.

Table 2. Vehicle and tyre parameters.

\begin{tabular}{|l||l|l|l|}
\hline Parameter & Physical Meaning & Unit & Value \\
\hline$M$ & total mass & $\mathrm{kg}$ & 1000 \\
$I z$ & yaw inertia & $\mathrm{kgm}^{2}$ & 1600 \\
$l_{f}$ & CG to front axle & $\mathrm{m}$ & 1.0 \\
$l_{r}$ & CG to rear axle & $\mathrm{m}$ & 1.8 \\
$\mathrm{w}$ & track width & $\mathrm{m}$ & 1.6 \\
$H G$ & mass centre hight & $\mathrm{m}$ & 0.35 \\
$I_{w}$ & nominal wheel rota- & $\mathrm{kgm}^{2}$ & 0.5 \\
& tional inertia & & \\
$R_{w}$ & loaded tyre radius & $\mathrm{m}$ & 0.3 \\
$\Lambda$ & ratio of front-to-rear & - & 0.5 \\
& suspension roll moment & & \\
$\rho$ & air mass density & $\mathrm{kg} / \mathrm{m}^{3}$ & 1.2 \\
$A_{\text {aero }}$ & reference area & $\mathrm{m}^{2}$ & 2.4 \\
$C_{D}$ & drag coefficient & - & 0.3 \\
$B, C, D, E$ & MF tyre coefficients & dimensionless & $0.7094,1.4097,1.000,0$ \\
\hline
\end{tabular}

\subsection{Modified Hamiltonian Algorithm}

Figure 5 shows a block diagram of the overall chassis control system. It includes a highlevel control reference generator (discussed in the next section) to provide a target CG acceleration demands for the vehicle. Then MHA acts as a control distributor, converting vehicle level demands into actuator signals. In [18], it was proposed to implement optimal control via terminal control cost, so that the Hamiltonian function becomes linear in the virtual controls, i.e. the vehicle force components and yaw moment. A single costate ratio (denoted by $\lambda$ in the following) determines the balance between yaw moment and mass centre force control. Inspired from this, the proposed method takes an alternative approach, by estimating this ratio in real-time using a single adaptation strategy.

The aim now is to convert acceleration demands, obtained from the particle model above, into actuator control signals: front wheel steering and four wheel braking torques. Let $\mathbf{a}^{d}=\left[a_{x}^{d}, a_{y}^{d}\right]^{T}$ be the desired mass centre acceleration vector, which we assume to be on the limits of friction. The Hamiltonian function is linear in the virtual controls

$$
H=p_{x} F_{x}+p_{y} F_{y}+\lambda M_{z}
$$




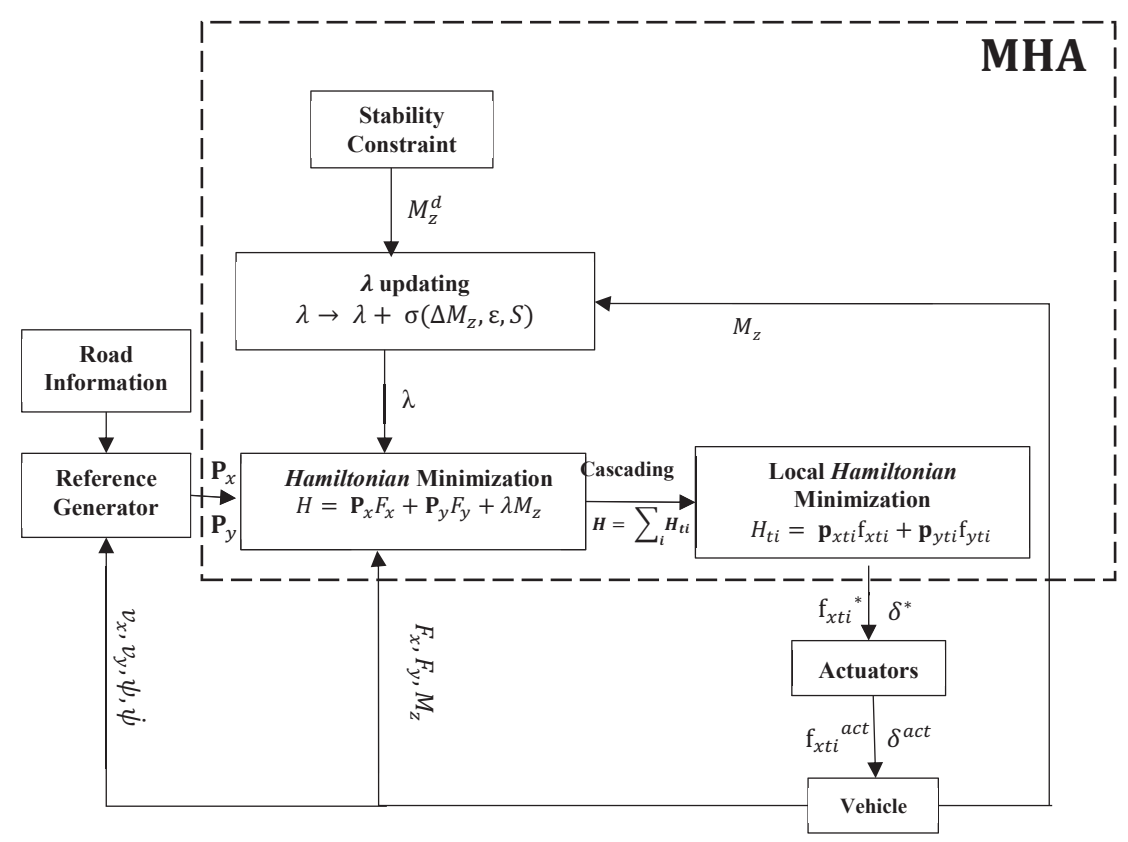

Figure 5. Overall structure of the MHA algorithm.

where $\mathbf{p}$ is a unit vector in the direction opposing the desired acceleration, $\mathbf{p}=-\mathbf{a}^{d} /\left|\mathbf{a}^{d}\right|$.

For yaw moment control, $\lambda$ is adapted to track a desired yaw moment $M_{z}^{d}$. In previous work [10-12] a switched adaptation rule was used

$$
\lambda \rightarrow \lambda+S \cdot \sigma\left(\Delta M_{z}\right)
$$

where $\sigma(x)=\operatorname{sgn}(x), \Delta M_{z}=M_{z}-M_{z}^{d}$, and $M_{z}$ is the estimated yaw moment from the controller, including contributions from longitudinal and lateral tyre forces. The controller is updated every $20 \mathrm{~ms}$ and the overall yaw moment tracking performance was found to be satisfactory [10-12]. Here, to suppress high-frequency oscillations, a saturation function is used for $\sigma$ in Equation 15:

$$
\sigma\left(\Delta M_{z}, \varepsilon\right)= \begin{cases}\varepsilon \Delta M_{z} & \text { if }\left|\varepsilon \Delta M_{z}\right|<1 \\ \operatorname{sgn}\left(\Delta M_{z}\right) & \text { otherwise }\end{cases}
$$

The following parameters were used: $\varepsilon=10^{-4}, S=0.1$. Since the Hamiltonian function is linear in tyre forces, the optimization can be achieved through a local minimization at each individual tyre. For more detail, the reader should refer to [10].

\section{Simulation Results}

To perform the simulation, a simple driver model is used to control the vehicle at a constant speed of $30 \mathrm{~m} / \mathrm{s}$ and tracking the original lane. All the parameters used for simulation are consistent with the sample case used for particle optimization. The collision avoidance is executed at $60 \mathrm{~m}$ (red car) to avoid a frontal obstacle located at $90 \mathrm{~m}$, illustrated via a pair of stationary blue vehicles, see Figure 6 . In this case $A=30 \mathrm{~m}$ for the particle-optimal avoidance strategy (see Figure 1) which is used as the acceleration 


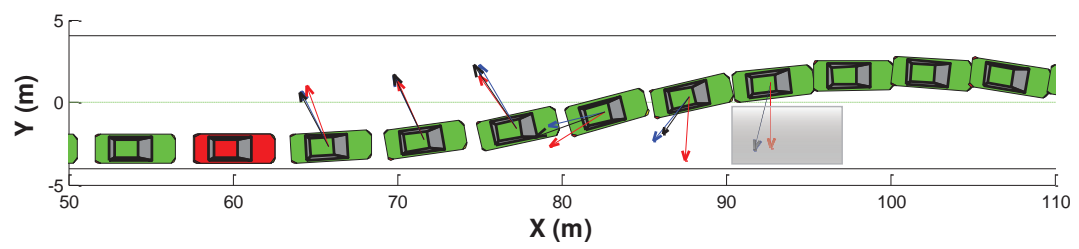

Figure 6. Vehicle trajectory and different level of acceleration vectors: 1) desired acceleration $\mathbf{a}^{d}$ (red arrow); 2) commanded acceleration $\mathbf{a}^{c m d}$ from MHA controller (black arrow); 3)actual vehicle CG acceleration $\mathbf{a}^{\text {act }}$ (blue arrow).

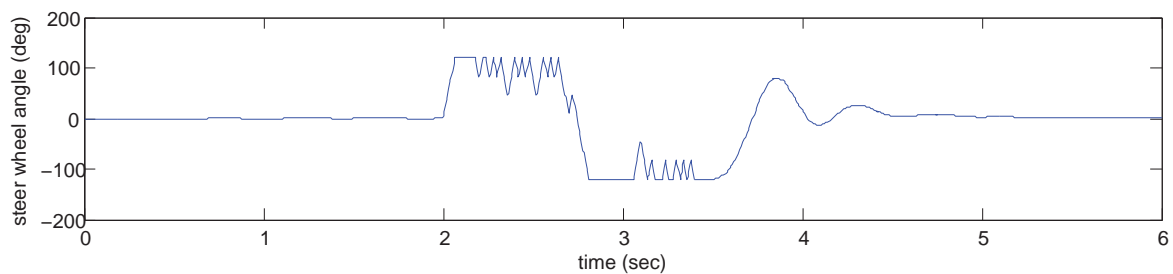

Figure 7. Steering angle output from the MHA controller (2 - 3.5 seconds) and driver model (other times).

reference. Taking account of the dimension of the obstacle and the subject vehicle, we formally require $\mathrm{B}=3.8 \mathrm{~m}$ for the $\mathrm{CG}$ trajectory. Figure 6 shows the path and acceleration during the interventions. Three acceleration vectors are plotted, also indicating the duration of the interventions. These are: (a) the desired acceleration reference $\mathbf{a}^{d}$ (red) obtained from particle optimization (section 2.2); (b) acceleration $\mathbf{a}^{\text {cmd }}$ expected from the MHA controller using its internal tyre model (black); (c) the actual vehicle CG acceleration vector $\mathbf{a}^{a c t}$ (blue). Differences between $\mathbf{a}^{d}$ and $\mathbf{a}^{\text {cmd }}$ arise due to actuator limitations, while difference between $\mathbf{a}^{c m d}$ and $\mathbf{a}^{a c t}$ results from the highly transient dynamics during the emergency lane change manoeuvre.

Discrepancies are most obvious during the transient phase in the middle of the emergency lane change manoeuvre when the rate of change of $\mathbf{a}^{d}$ is large. However, the three arrows converge well during the event. It appears that the tyre force control is satisfactory and the collision can be avoided while having minimum off-shoot. From Figure 6, it also can be seen that the maximum off-shoot is reached around $100 \mathrm{~m}$ along the track. Compared to other strategies, the maximum off-shoot is significantly reduced from around $3.7 \mathrm{~m}$ to $1.2 \mathrm{~m}$. Figure 7 shows the steering wheel angle output from the MHA controller during the event. It is seen that, the MHA controller kept adjusting the steering wheel angle output at each updating time step during the event and working within saturation limits of +- 120 degrees. The oscillation of the steering input can be further reduced by using a smooth switching function within the controller, for more details see [10].

Figure 8 shows that by dynamically adapting $\lambda$, it is possible to control yaw moments and actually achieve tight control of body sideslip. It is worth mentioning that, for the stability control, the aim is not to minimize the body sideslip angle, but rather control the body sideslip angle within a suitable range to facilitate lateral force generation. Here, the upper threshold on slip angle is chosen to be $6^{\circ}$ and the actual peak is within $\pm 6^{\circ}$ shown in Figure 9. The effect of the tyre relaxation and vehicle inertia during transient state is also captured in Figure 8. At around 2.7 seconds, the desired yaw moment $M_{z}^{d}$ starts to increase; this is to prevent excessive over-shoot during the second phase lane keeping control. And the actual yaw moment $\left(I_{z} \ddot{\psi}\right)$ follows the commanded yaw moment $M_{z}$ based on $\lambda$ adaptation strategy, but with some off-set value. However, the whole process only lasts around 0.3 seconds and the overall yaw tracking performance is highly 


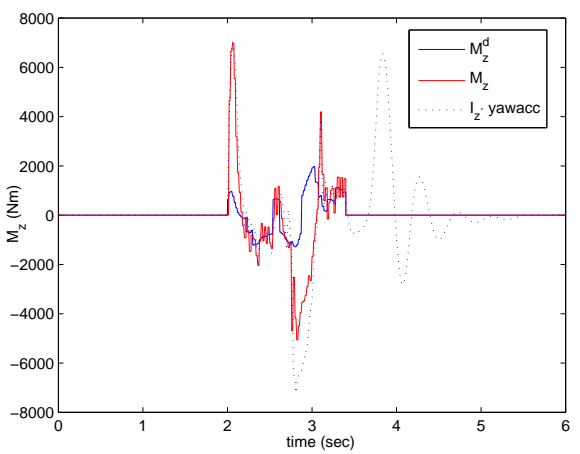

Figure 8. Yaw moment tracking: desired yaw moment $M_{z}^{d}$; commanded yaw moment $M_{z}$; actual yaw moment $I_{z} \cdot \ddot{\psi}$
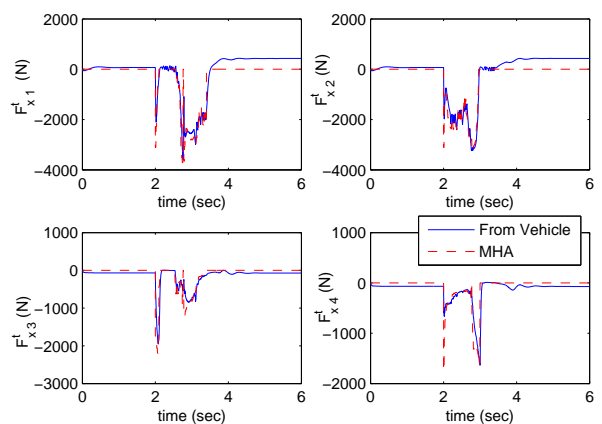

Figure 10. Commanded braking forces vs. actual braking forces.

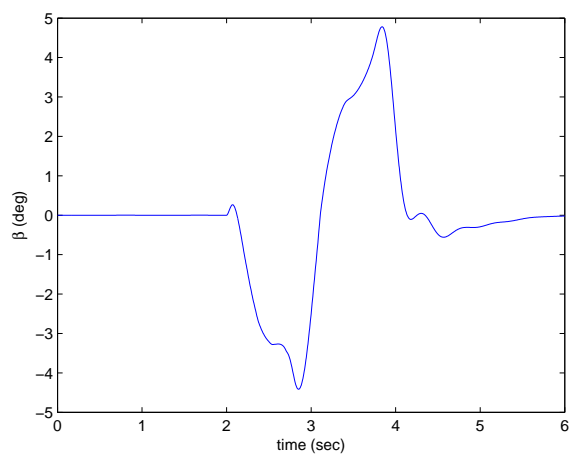

Figure 9. Vehicle body sideslip angle profile.
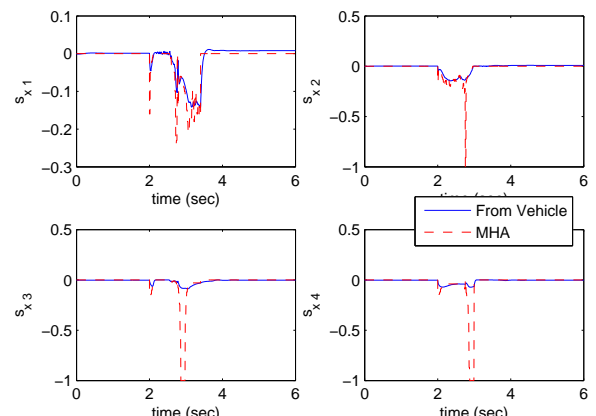

Figure 11. Commanded slip ratio vs. actual slip ratio.

satisfactory. This off-set between the commanded and actually yaw moment can also be explained with Figure 10 - 13. During 2.7 - 3 seconds, the actual braking forces at the rear tyres are less than the commanded braking forces. This is because the force allocation formulation presented above operates under the assumption of perfect estimation of the effective friction level on each tyre, which is clearly a source of error since both tyre loads and vehicle dynamics are subject to rapid change as the vehicle is making a sudden lane change. Hence it is expected that with a slip regulation scheme, the controller would be capable of allocating the braking forces more precisely.

\section{Conclusion}

A particle model is used to calculate the optimal solution of a collision avoidance problem with the focus on minimizing the resultant over-shoot. The optimal control shows a rotation of the global acceleration vector at a given set of collision avoidance scenario. As a special case, it is found that, at the limiting braking distance, the acceleration vector is fixed in the global frame. Then different collision avoidance strategies, represented with different acceleration reference are compared in terms of a trade-off between the braking distance and resulting over-shoot in the collision avoidance scenario. It is found that, using a combined braking and steering strategy resulting from minimizing the lateral velocity component at the collision avoidance location presents the least amount of overshoot in the lane keeping phase. A recently developed efficient control allocation method 

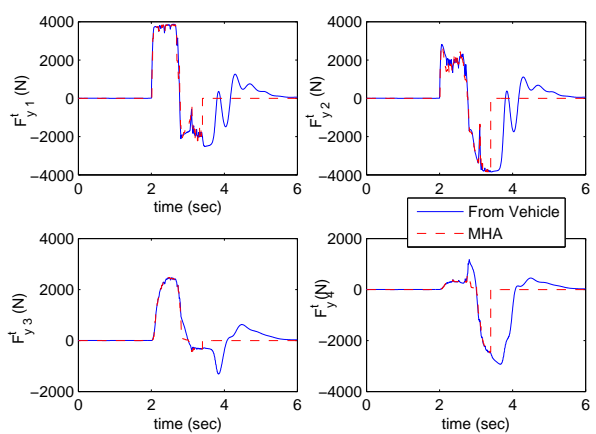

Figure 12. Commanded lateral tyre forces vs. actual lateral tyre forces.
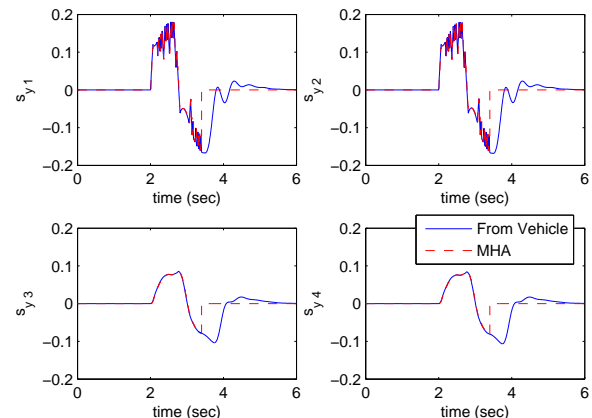

Figure 13. Commanded slip angle vs. actual slip angle.

MHA is applied to operate the collision avoidance manoeuvre at the limit of friction in the fully autonomous fashion (automatic braking and steering), given the acceleration reference provided with an ideal particle model. The simulation results have shown a close match with the ideal solution from a particle model while the vehicle stability is controlled via closely tracking a desired yaw reference. This is achieved through distributing the braking torque as precisely as possible. Although simulation has shown some off-set between the commanded and actual braking force for a short period of time during the intervention, it is expected this can be improved by introducing a low-level slip ratio controller. It is also worth mentioning that, the proposed control strategy can be easily extended to include driving torque (torque vectoring) e.g. via active differentials or electric motors. Such a control method might be used for intersection safety with regards to the possibility of crossing the intersection ahead of an oncoming vehicle for collision avoidance. It will be interesting to see, with the added control authority through driving torque, how much can be gained.

The MHA method is designed to be executed in real-time and has been tested previously with an experimental vehicle for developing a departure prevention system. The experimental results have shown a high level of correlation with the simulation results. Regarding collision avoidance experiment, the time delay in the system should be compensated in advance for better control performance, and this can potentially be achieved through the particle reference. Additionally, in real-world applications an updating acceleration reference based on the formulation of the Appendix A could be obtained, for example, using a pre-stored lookup table relating parameters $k_{i}$ to the updating geometric parameters of the collision avoidance problem. Another possible application would be to adapt to variations in road surface friction.

\section{Appendix A. Optimal Control Proof}

Write Equations 3 in the state-space form

$$
\underline{\dot{x}}=\mathbf{A} \underline{\mathbf{x}}+\mathbf{q}(\underline{\mathbf{u}})
$$

where the state vector $\underline{\mathbf{x}}$ and input vector $\underline{\mathbf{u}}$ are given

$$
\begin{aligned}
& \underline{\mathbf{x}}=\left[\begin{array}{llll}
x_{1} & x_{2} & x_{3} & x_{4}
\end{array}\right]^{\mathrm{T}} \\
& \underline{\mathbf{u}}=\left[\begin{array}{ll}
u_{1} & u_{2}
\end{array}\right]^{\mathrm{T}}
\end{aligned}
$$


where $x_{1}=X_{E}, x_{2}=\dot{X}_{E}, x_{3}=Y_{E}, x_{4}=\dot{Y}_{E}$, subject to friction constraint $u_{1}^{2}+u_{2}^{2} \leq \mu^{2} g^{2}$

The optimal control problem is formulated as minimize the lateral off-tracking $\Delta y$ after the collision avoidance, as discussed above in section 2.2 , equivalent to minimize lateral velocity component $\left(\dot{Y}_{E}\right)$ at $\mathrm{P}_{2}$. This results in minimizing the terminal cost. The terminal time $\mathrm{T}$ for the first phase control is stated in section 2.2 as $t_{1}$.

$$
\Phi(\underline{\mathbf{x}})=x_{4}
$$

The optimal control problem for the particle model is solved by extending the dynamic model to an augmented Hamiltonian system

$$
\underline{\dot{x}}=\frac{\partial H}{\partial \underline{P}} \quad \underline{\dot{P}}=-\frac{\partial H}{\partial \underline{\mathbf{x}}}
$$

where $\underline{P}$ is the co-state vector and where the Hamiltonian function is:

$$
H=\underline{P}^{\mathrm{T}} \cdot[\mathbf{A} \underline{\mathbf{x}}+\mathbf{q}(\underline{\mathbf{u}})]
$$

According to the Pontryagin minimum principle, the optimal control problem is now reduced to minimize the Hamiltonian function over the set of all permissible controls $\underline{\mathbf{u}}$. This is a free terminal time problem, $H(\mathrm{t})=0$ during the whole time history. According to Equation A4, A5 The equations for the co-states are particular simple:

$$
\underline{\dot{P}}=-\frac{\partial H}{\partial \underline{\mathbf{x}}} \Longrightarrow \underline{\dot{P}}=\left[0,-\mathrm{P}_{1}, 0,-P_{3}\right]^{\mathrm{T}}
$$

These relations are easily integrated to yield:

$$
\underline{P}=\left[-k_{1}, k_{1} t+k_{2},-k_{3}, k_{3} t+k_{4}\right]^{\mathrm{T}}
$$

With only terminal cost function, $L=0$ the Hamiltonian function of the system is

$$
H=P_{1} x_{2}+P_{2} u_{1}+P_{3} x_{4}+P_{4} u_{2}
$$

Thus the optimal control for the acceleration reference is

$$
\tan \theta^{*}(t)=\frac{P_{4}}{P_{2}}=\frac{k_{2} t+k_{4}}{k_{1} t+k_{2}}
$$

We do not care about the final value of the longitudinal speed at $t=\mathrm{T}$, resulting in $P_{2}(\mathrm{~T})=0$ and the transversality condition is $P_{4}(\mathrm{~T})=-(\partial \Phi / \partial \underline{\mathbf{x}})_{\mathrm{T}}=-1$. The boundary conditions are

$$
\left(\begin{array}{l}
x_{1}(0) \\
x_{2}(0) \\
x_{3}(0) \\
x_{4}(0)
\end{array}\right)=\left(\begin{array}{c}
0 \\
v_{0} \\
0 \\
0
\end{array}\right) \quad\left(\begin{array}{l}
x_{1}(\mathrm{~T}) \\
P_{2}(\mathrm{~T}) \\
x_{3}(\mathrm{~T}) \\
P_{4}(\mathrm{~T})
\end{array}\right)=\left(\begin{array}{c}
A \\
0 \\
B \\
-1
\end{array}\right)
$$

Equation A4 combined with the above initial conditions, the optimal solution can be solved numerically with MATLAB built-in function bvp4c. The following gives some 
analytical aspects of the optimal solution.

Considering the optimality condition

$$
H=P_{2} u_{1}+P_{4} u_{2}+H_{0}=\underline{\hat{P}} \cdot \underline{\mathbf{u}}+H_{0}
$$

where $H_{0}$ is independent of $u_{i}$, the optimal control $\underline{\mathbf{u}}^{*}$

$$
\underline{\mathbf{u}}^{*}=-\mu g \underline{\hat{P}}
$$

where $\underline{\hat{P}}=\frac{\left[P_{2} P_{4}\right]^{\mathrm{T}}}{P_{2}^{2}+P_{4}^{2}}$. Note that, for the free terminal time problem, $H(t)$ stays zero over the entire control trajectory

$$
H(0)=P_{1}(0) v_{0}+\left(\begin{array}{l}
P_{2}(0) \\
P_{4}(0)
\end{array}\right) \cdot\left(\begin{array}{l}
u_{1}(0) \\
u_{2}(0)
\end{array}\right)+0=0
$$

where

$$
\left(\begin{array}{l}
u_{1} \\
u_{2}
\end{array}\right)=-\mu g\left(\begin{array}{l}
P_{2} \\
P_{4}
\end{array}\right) \times \frac{1}{\sqrt{P_{2}^{2}+P_{4}^{2}}}
$$

and

$$
\left(\begin{array}{l}
P_{2} \\
P_{4}
\end{array}\right) \cdot\left(\begin{array}{l}
u_{1} \\
u_{2}
\end{array}\right)=-\mu g \frac{P_{2}^{2}+P_{4}^{2}}{\sqrt{P_{2}^{2}+P_{4}^{2}}}=-\mu g \sqrt{P_{2}^{2}+P_{4}^{2}}
$$

So at $t=0$

$$
H(0)=-k_{1} v_{0}-\mu g \sqrt{k_{2}^{2}+k_{4}^{2}}=0
$$

and

$$
k_{1}=\frac{-\mu g \sqrt{k_{2}^{2}+k_{4}^{2}}}{v_{0}}
$$

and

$$
\mathrm{T}=-\frac{k_{2}}{k_{1}}=\frac{k_{2} v_{0}}{\mu g \sqrt{k_{2}^{2}+k_{4}^{2}}}
$$

\section{References}

[1] Edwin L Baldwin. Collision avoidance warning system, October 6 1981. US Patent 4,293,857.

[2] Avner Ben-Yaacov, Masha Maltz, and David Shinar. Effects of an in-vehicle collision avoidance warning system on short-and long-term driving performance. Human Factors, 44(2):335-342, 2002.

[3] Wassim G Najm, Mary D Stearns, Heidi Howarth, Jonathan Koopmann, and John Hitz. Evaluation of an automotive rear-end collision avoidance system. Technical report, 2006.

[4] Ayumu Doi, Tetsuro Butsuen, Tadayuki Niibe, Takeshi Takagi, Yasunori Yamamoto, and Hirofumi Seni. Development of a rear-end collision avoidance system with automatic brake control. JSAE Review, 15(4):335-340, 1994. 
[5] Joseph Funke, Matthew Brown, Stephen M Erlien, and J Christian Gerdes. Collision avoidance and stabilization for autonomous vehicles in emergency scenarios. IEEE Transactions on Control Systems Technology, 25(4):1204-1216, 2017.

[6] Kai-Tai Song, Chih-Hao Chen, and Cheng-Hsien Chiu Huang. Design and experimental study of an ultrasonic sensor system for lateral collision avoidance at low speeds. In Intelligent Vehicles Symposium, 2004 IEEE, pages 647-652. IEEE, 2004.

[7] Jonas Jansson. Collision Avoidance Theory: With application to automotive collision mitigation. $\mathrm{PhD}$ thesis, Linköping University Electronic Press, 2005.

[8] Andrew Gray, Mohammad Ali, Yiqi Gao, J Hedrick, and Francesco Borrelli. Semi-autonomous vehicle control for road departure and obstacle avoidance. IFAC control of transportation systems, pages 1-6, 2012.

[9] Shinichiro Horiuchi, Ryusuke Hirao, Kazuyuki Okada, and Shinya Nohtomi. Optimal steering and braking control in emergency obstacle avoidance. Nippon Kikai Gakkai Ronbunshu C Hen(Transactions of the Japan Society of Mechanical Engineers Part C)(Japan), 18(10):3250-3255, 2006.

[10] Yangyan Gao, Mathias Lidberg, and Timothy Gordon. Modified hamiltonian algorithm for optimal lane change with application to collision avoidance. MM Science Journal MAR, pages 576-584, 2015.

[11] Yangyan Gao, Timothy Gordon, and Mathias Lidberg. Implementation of a modified hamiltonian algorithm for control allocation. In Advanced Vehicle Control: Proceedings of the 13th International Symposium on Advanced Vehicle Control (AVEC'16), September 13-16, 2016, Munich, Germany, page 157. CRC Press, 2016.

[12] Yangyan Gao, Tim Gordon, Mathias Lidberg, and Matthijs Klomp. An autonomous safety system for road departure prevention based on combined path and sideslip control. In The Dynamics of Vehicles on Roads and Tracks: Proceedings of the 24th Symposium of the International Association for Vehicle System Dynamics (IAVSD 2015), Graz, Austria, 17-21 August 2015, page 281. CRC Press, 2016.

[13] Arthur Earl Bryson. Applied optimal control: optimization, estimation and control. CRC Press, 1975.

[14] Lawrence F Shampine, Jacek Kierzenka, and Mark W Reichelt. Solving boundary value problems for ordinary differential equations in matlab with bvp4c. Tutorial notes, 2000:1-27, 2000.

[15] William F Milliken, Douglas L Milliken, et al. Race car vehicle dynamics, volume 400. Society of Automotive Engineers Warrendale, 1995.

[16] Hans B Pacejka and Egbert Bakker. The magic formula tyre model. Vehicle system dynamics, 21(S1):1-18, 1992.

[17] TJ Gordon and Matt C Best. On the synthesis of driver inputs for the simulation of closed-loop handling manoeuvres. International Journal of Vehicle Design, 40(1-3):52-76, 2005.

[18] Derong Yang, Timothy J Gordon, Bengt Jacobson, and Mats Jonasson. Quasi-linear optimal path controller applied to post impact vehicle dynamics. IEEE transactions on intelligent transportation systems, 13(4):1586-1598, 2012. 